\section{Laboratory approach for detection of non-invasive fungal rhinosinusitis: A case-control study}

Nissreen E. ELBadawy', Takwa E. Meawed ${ }^{1}$ and Mohammad Waheed El-Anwar ${ }^{2}$

\section{Abstract}

Objective: Noninvasive fungal rhinosinustis (NFRS) is a problematic clinical condition due to its resistant to traditional medical treatment. Surgical debridement is the basis for its treatment. Mycology laboratory investigation can help to solve this issue. This case control study was designed to investigate the frequency of NFRS in our locality and identify fungal species incriminated in this clinical condition at the Otorhinolaryngology department, Zagazig University Hospitals. The study evaluated the role of microscopic examination, antigen detection and PCR in comparison to culture methods used in diagnosis of fungal agents.

Materials: Sinus materials were collected from 78 cases which have been clinically and radiologically confirmed with NFRS over the period June 2013 to September 2015. A control group 78 subjects were included with healthy sinuses from whom nasal smears were obtained. Samples were processed in the Mycology Laboratory, and all specimens were examined microscopically in $10 \% \mathrm{KOH}$ preparations. Lactophenol cotton blue slide preparations were used to detect microscopic structures of hyphae and conidia. PCR amplification of the extracted DNA was performed using fungal universal primers for amplification of $28 \mathrm{~S}$ rDNA genes.

Results: Microscopic examinations revealed hyphae and fruiting bodies in $37(47.4 \%)$ of the cases. Fungal culture detected 36 patients infected with NFRS. Aspergillus fumigatus was the most frequently isolate from fungal rhinosinusitis (52.7 \%) of the cases, followed by Penicillium spp. (22.2\%). PCR amplification exhibits the same sensitivity and specificity as those demonstrated by microscopic examination (100\% and 97.3\%,
Corresponding author:

Dr. Takwa El Sayed Meawed

” takwa_farid@hotmail.com 
respectively). ELISA of Aspergillus galactomannan (GM) antigen lacked sensitivity (58.3\%), while specificity was high (100\%).

Conclusion: This study demonstrates that proper mycological investigation using direct microscopic examination and culture are necessary to confirm presence of noninvasive fungal rhinosinustis in patients.

Key words: Rhinosinusitis; fungus; $\mathrm{KOH}$; galactomannan; $P C R$

\section{Introduction}

Non-invasive fungal rhinosinusitis (NFRS) presented with sinus fungal ball is a common disorders affecting immunocompetent individuals of all age groups. The incidence of NFRS has increased significantly in many countries possibly due to air pollution, allergy, and climate changes $[1,2]$. Sinus fungal ball is associated clinically by nasal congestion, purulent or bloody discharge, headache, and impaired smell sensation, which are non-conclusive for diagnosis, and may be misdiagnosed as soft-tissue tumors [35]. Available studies indicate that Aspergillus spp. is the most common etiological agents for NFRS [6]. Surgical removal of the affected sinus mucosa is the mainstay for treatment of almost all cases with reestablishing the drainage from the affected sinus. There is no need for antifungal therapy, except in cases of allergic association or complication with bone erosion [7,8]. Microbiology Laboratory can help in confirmation of the diagnosis and verification of the etiological fungal agent [9].

Mycology culture is still considered the gold standard despite of being an old traditional diagnostic technique. Culture practice should not be ignored, even with delay in fungal identification [10, 11]. Non-molecular markers are available alternative tools supporting the diagnosis of fungal infection in combination with clinical, radiologic, and other microbiological criteria [12].

PCR is a useful method that detects viable and nonviable fungal pathogens and includes a variety of protocols. Some protocols use pan fungal primer PCR assays, whereas single species detection can be carried out using species specific primers [13]. Mycology laboratory investigation followed by surgical debridement would prevent usage of unnecessary antifungal therapy in NFRS.

According to our best knowledge, data about incidence and etiology of NFRS in Egypt is not satisfactory. This study was planned to determine the frequency of NFRS in our locality at Zagazig University Hospitals, using microscopic examination, antigen detection and PCR as compared to fungal culture.

\section{Materials and Methods}

\section{Setting and study design}

A case-control study was carried out in Otorhinolaryngology Department, Zagazig University Hospi- 
tals in association with the Mycology Lab, Medical Microbiology and Immunology Department, Faculty of Medicine, Zagazig University, over a period June 2013 to September 2015. An informed written consent was obtained from all investigated subjects as well as the approval of the Institutional Board Review of Zagazig University Hospitals.

\section{Subjects and clinical Samples}

A total of 78 clinical samples were collected from clinical case group with suspected NFRS. Paranasal sinus tissue specimens were collected with the following inclusion criteria; CT radiological findings as soft tissue masses with partial or complete opacification of a sinus with hyper dense areas (mottling) without bone invasion, intraoperative characteristic muddy discharge with nasal polyps. Exclusion criteria include the following: Any immunodeficiency condition to rule out invasive or opportunistic fungal infection, no previous treatment with oral, parenteral corticosteroids or antifungal agents. The second (control) group included 78 subjects with apparently healthy sinus (patients who were admitted for septoplasty and/or rhinoplasty).

\section{Specimens Collection}

Paranasal sinus tissue specimens were collected from the clinical case group in the operating room [14]. A povidoniodine solution was used for antisepsis of nostrils [5]. Regarding control group, nasal smears were obtained and examined with light microscopy and fungal cultivation.

Surgical specimens of sinus mass were obtained in sterile screw-capped containers containing Sabouraud's dextrose broth. Clinical data was collected and analyzed. Specimens were processed in safety cabinet of the Mycology Lab.

\section{Microscopic Examination and Cultivation}

All surgically excised specimens and nasal smears placed in Sabouraud's dextrose broth were centrifuged with glass beads. A portion of the pellet was subjected to $10 \%$ potassium hydroxide $(\mathrm{KOH})$ wet mount preparation and was examined by $40 \times$ objectives for the presence of fungal elements [15].

The remaining portion of the pellet was inoculated on two plates of Sabouraud's dextrose agar (SDA) containing gentamicin (20 mg/liter) and chloramphenicol (50 mg/liter) and Sabouraud's brain heart infusion agar (SBAHI) [16]. One plate from both media was incubated at $37^{\circ} \mathrm{C}$, and the other one at the room temperature. Culture plates were observed every two days for fungal growth and up to 6 weeks before considering them negative. Growth was recognized by gross examination of aerial mycelium, color and growth rate. Slide culture technique was performed with Lactophenol cotton blue mount, to reveal fungal structure of the mycelium, conidial types and hyphae which are characteristics for each species [16].

\section{Fungal antigen detection}

Aspergillus Galactomannan (GM) was examined in supernatant of the processed tissue specimens with Platelia Aspergillus kit (England, UK) according to the manufacture instructions. Briefly, it is a sandwich immunoenzymatic technique depends on detection of Aspergillus Galactomannan using rat monoclonal antibody EBA2.

\section{DNA extraction and PCR}

A loopful of fresh tissue was suspended in $200 \mu \mathrm{l}$ of sterile water, and DNA extraction was performed by QIAmp tissue DNA extraction kit (QIAGEN, United Kingdom) according to the procedure described by the manufacture.

PCR of DNA extract was performed using universal fungal primers for the 28S rDNA forward primer [5'-GTG AAA TTG TTG AAA GGG AA-3'] and reverse [5'-GAC TCC TTG GTC CGT GTT-3'] (Ebersdorf, Germany) as recommended [17]. PCR amplifications reaction was carried out in $25-\mu$ l reaction volumes with a Biometra thermocycler (Biometra, Germany). All PCR runs included a positive control with DNA 
of $A$. fumigatus, and two negative controls with PCR buffer. Cycling conditions were as follows: initial denaturation at $95^{\circ} \mathrm{C}$ for 10 min followed by 49 cycles of denaturation at $95^{\circ} \mathrm{C}$ for $1 \mathrm{~min}$, annealing at $50^{\circ} \mathrm{C}$ for $1 \mathrm{~min}$, and extension at $72^{\circ} \mathrm{C}$ for 2 min followed by a final extension at $72^{\circ} \mathrm{C}$ for $10 \mathrm{~min}$. Amplification products were separated and analyzed by electrophoresis in a $2 \%$ agarose gel with ethidium bromide stain. PCR products (260 bp) in length were interpreted as an evidence of fungal DNA.

\section{Statistical analysis}

Collected Data were tabulated and analyzed using IBM SPSS version19, and the results of two groups were compared. Regarding case group, symptoms of patients with positive and negative fungal cultivation were compared. Validity tests and Chi-square test were applied in statistical analysis.

\section{Results}

Out of 78 cases enrolled in this study, 36 cases (46.1\%) were confirmed to be associated with NFRS using culture media. No mycological evidence of FRS was found in the remaining 42 patients. The frequency of positive sinus NFRS was 18 (50\%) in both males and females, and was more frequent in middle age patients with Mean \pm SD of $33.3 \pm 9.4$ years.
Culture method has diagnosed 36 cases of NFRS. Aspergillus fumigatus was the most frequent fungal species isolated from FRS cases with statistically significant difference $(P<0.001)$. It was recovered from 19 (52.7 \%) of NFRS cases, followed by Penicillium spp. in 8 (22.2\%). Only $3(8.3 \%)$ positive fungal cultures were recovered from the healthy control group (Table 1). Microscopic examination by $10 \%$ $\mathrm{KOH}$ revealed the presence of hyphae in 33 (42.3\%) of the cases), hyphae with fruiting bodies and conidia in 4 cases (5.1\%) of samples.

Antigen ELISA revealed the presence of Aspergillus galactomannan in 21/78 (26.9 \%) in association with chronic sinusitis cases. Of these 18 cases with were positive for Aspergillus fumigatus culture. Three false positive ELISA cases were identified as Scopulropsis by culture. Negative assay results were noticed in 15 cases of positive fungal culture. The remaining 42 cases were negative by both Antigen ELISA and fungal cultures.

PCR amplification was validated first by the presence of fungal $28 \mathrm{~S}$ rDNA of $260 \mathrm{bp}$ using positive control strain of Aspergillus fumigatus. Positive fungal DNA was demonstrated in $37 / 78$ ( $47.4 \%$ ) of clinically suspected cases, of these $\mathbf{3 6 / 7 8}(\mathbf{4 6 . 2 \% )}$ were confirmed by positive fungal cultures. Aspergillus antigen detection method showed high specificity and the lowest sensitivity (58.3\%). Performance comparison of the four used techniques for diagnosis of FRS is shown in Table $\mathbf{3}$.

Table 1. Fungal species isolates from non-invasive rhinosinusitis patients and healthy control

\begin{tabular}{|c|c|c|c|}
\hline Fungus species & $\begin{array}{l}\text { Case group } \\
\text { Total no.36(\%) }\end{array}$ & $\begin{array}{l}\text { Healthy control } \\
\text { Total no. (\%) }\end{array}$ & $\begin{array}{c}P \\
\text { value }\end{array}$ \\
\hline Aspergillus fumigatus & $19(52.7)$ & $1(33.3)$ & $<0.001$ \\
\hline Penicillium spp. & $8(22.2)$ & $2(66.7)$ & 0.007 \\
\hline Scopulariopsis spp. & $3(8.3)$ & $0(0.0)$ & 0.014 \\
\hline Aspergillus niger & $2(5.5)$ & $0(0.0)$ & 0.046 \\
\hline Alternaria spp. & $2(5.5)$ & $0(0.0)$ & 0.046 \\
\hline Cladosporium spp. & $1(2.7)$ & $0(0.0)$ & 0.157 \\
\hline Aurobasidium spp. & $1(2.7)$ & $0(0.0)$ & 0.157 \\
\hline Total & $36(100.0)$ & $3(100.0)$ & \\
\hline
\end{tabular}




\section{Discussion}

The incidence of Non-invasive fungal rhinosinusitis (NFRS) increased markedly over the past three decades due both to increased fungal pollution in the environment and improved diagnostic modalities $[8,18,19]$. Different prevalence rates of fungal species causing rhinosinusitis are related to the geographic and seasonal variations as have been reported in India and Germany $[20,21]$. To our best knowledge, NFRS is not being extensively discussed as a separate clinical entity in Arabian countries. A combined phenotypic and molecular methods are currently the best approach for fungal identification, especially for moulds [9].

This study revealed that aspergillosis was diagnosed in more than half of cases (58.2\%), with Aspergillus fumigatus being the most predominant isolate. Several studies from India and other countries found NFRS mostly associated with aspergillosis $(63.1 \%$ and $70 \%)$, respectively $[8,22]$. Whereas, Aspergillus flavus followed by Aspergillus fumigatus are being the most common etiological agents of NFRS, and were responsible for $76.19 \%$ and $88 \%$ of cases in two separate studies in India [23, 24].

This study detected that Penicillium spp. accounted for $22.2 \%$ of the positive cases. This result is similar to a study done in Crete/Greece where
$28.1 \%$ of NFRS cases caused by Penicillium spp. [25], while Cladosporium spp. was less detected (one case) in our study and other studies in Greece and Iran $[25,26]$. Additionally, our study found only 2 (5.5\%) cases of Alternaria spp, whereas this fungus was recovered from $75 \%$ and 9.7 of the positive cases in Iran and Greece, respectively $[25,27]$. The study of Buzina et al. [28] in Germany, reported that Cladosporium spp., Alternaria spp. and Aureobasidium pullulans showed a significantly higher occurrence during late summer and early autumn.

In this study, scopoularisis was recovered from 3 cases $(8.3 \%)$, and both candidiasis and zygomycosis were not recovered from any patient in contrast to reported studies [25].

Chatterjee and Chakrabarti reported that epidemiology of fungal rhinosinusitis is still not satisfactory, and its classification is still confusing, each of the clinicopathological variants of FRS is associated with exclusive geographical and host related risk factors, and different fungal etiological agents [29].

All cases included in this study were diagnosed to have NFRS, and these were mostly occurred in immunocompetent patients. Additionally, the most prevalent clinical presentation associated with NFRS was nasal obstruction (100\%) (Table 2). This result is in agreement with other researchers who reported the same result [22].

Table 2. Clinical presentations of non-invasive rhinosinusitis case group in association with culture results

\begin{tabular}{|c|c|c|c|}
\hline & \multicolumn{2}{|c|}{ Culture results } & \multirow[b]{2}{*}{ P value } \\
\hline & $\begin{array}{l}\text { Positive cases } \\
\text { Total no. } 36(\%)\end{array}$ & $\begin{array}{l}\text { Negative cases } \\
\text { Total no.42(\%) }\end{array}$ & \\
\hline Nasal obstruction & $36(100)$ & $23(54.7)$ & 0.017 \\
\hline Nasal discharge & $24(66.6)$ & $32(76.1)$ & 0.131 \\
\hline Facial pain & $24(66.6)$ & $38(90.4)$ & 0.012 \\
\hline Headache & $18(50)$ & $34(80.9)$ & 0.002 \\
\hline Rhinorrhea & $6(16.6)$ & $26(61.9)$ & $<0.001$ \\
\hline Epiphora & $6(16.6)$ & 33 (78.5) & $<0.001$ \\
\hline Snoring & 6 (16.6) & $27(64.2)$ & $<0.001$ \\
\hline
\end{tabular}


Table 3. Comparison of microscopic examination, antigen detection, and PCR methods used for diagnosis of 78 fungal rhinosinusitis cases in relation to performing culture

\begin{tabular}{|l|c|c|c|c|c|c|}
\hline & Sensitivity & Specificity & $\begin{array}{c}\text { Positive } \\
\text { predictive value }\end{array}$ & $\begin{array}{c}\text { Negative } \\
\text { predictive value }\end{array}$ & Accuracy \\
\hline Micscopic examiunaon & $100 \%$ & $97.6 \%$ & $97.3 \%$ & $100 \%$ & $98.7 \%$ \\
\hline Antigen detection & $58.3 \%$ & $100 \%$ & $100 \%$ & $73.7 \%$ & $80.9 \%$ \\
\hline PCR & $100 \%$ & $97.6 \%$ & $97.3 \%$ & $100 \%$ & 9.601 \\
\hline
\end{tabular}

This study confirmed the highest sensitivity of microscopic examination (100\%), and specificity of $97.6 \%$, compared to the study of Lacroix and his colleagues who reported sensitivity and specificity of $73 \%$ and $100 \%$; respectively [30]. In addition, microscopy revealed an extra positive case over the culture diagnosis, and the same observation was documented by the study of Broglie et al. [31]. However, a difference between microscopy and culture results could be achieved by using multiple culture media for detection of fungal-like mucin $[32,33]$.

The results of our fungal detection by PCR showed $100 \%$ sensitivity and $97.6 \%$ specificity as compared with standard culture techniques used. Similar results were reported by Polzehl et al.[21], since their study demonstrated that $61 / 77$ of sinus lavage samples were positive for Aspergillus spp. in both culture and Aspergillus-specific nested PCR.

The present study showed that galactomannan (GM) antigen ELISA test has $58.3 \%$ sensitivity, $100 \%$ specificity and $80.8 \%$ accuracy, while a study of Kostamo et al. [34] showed that all FRS positive samples were negative by GM antigen ELISA test, and this test gave false positive results in five samples, that were negative by both culture method and PCR. Therefore, we could conclude that GM ELISA is not reliable in diagnosing Aspergillus infection of the paranasal sinuses.
It has been reported that there is an association between sinusitis and elevated mould-specific lgGlevels for Aspergillus fumigatus, Aspergillus versicolor, Aureobasidium pullulans [32,34], and it has also been demonstrated that cross-reactivity exist between cell wall components of Scopulariopsis brevicaulis and Aspergillus spp. [35]. This study also confirm that galactomannan antigen test is not specific for detection filamentous fungi and can't differentiate between Aspergillus, Penicillium and Scopulariopsis. In conclusion, Aspergillus fumigatus was the most frequent species isolated from NFRS cases, and both direct microscopic examination and PCR method have nearly the same sensitivity and specificity in detection the fungal agents. Surgical debridement was mandatory to remove fungal elements.

\section{Acknowledgement}

The authors are grateful to the rewarding advices and technical assistance of Professor Magda Mostafa Azab, Professor and head of Medical Microbiology \& Immunology, Faculty of Medicine, Zagazig University, Egypt. Great thanks to Professor Wafaa Ahmed Zahran, Professor of Medical Microbiology \& Immunology and Vice-dean, Faculty of Medicine, Menofia University for her fruitful assistance in reviewing this work. 


\section{References}

1. Zhu H, Zhang W, Guan J, Ye J, Su K. CT imaging and clinical features of sinus fungus ball with bone erosion. JNSCI 2015; 1(4): e69.

2. Ragab A, Samaka RM, Salem M. Impact of fungal load on diagnosis and outcome of allergic fungal rhinosinusitis. Eur Arch Otorhinolaryngol. 2014; 271(1):93-101.

3. Deshmukh J, Bhise S, Band A. Mycological Study of Aspergillus Infections in Chronic Paranasal Sinusitis in Eastern Maharashtra: A Longitudinal Study. IJSS 2014; 2 (7): 53-58.

4. Thrasher RD, Kingdom TT. Fungal infections of the head and neck: An update. Otolaryngol Clin North Am. 2003; 36:577-94

5. Palacios E, Jones W, Alvernia J. Sinonasal mycetoma. Ear Nose Throat J. $2008 ; 87(11): 606-8$

6. Soontrapa P, Larbcharoensub N, Luxameechanporn $T$, et al. Fungal rhinosinusitis: a retrospective analysis of clinicopathologic features and treatment outcomes at Ramathibodi Hospital. SE ASIAN J TROP MED; 41(2):442-9.

7. Aribandi M, McCoy V, Bazan, C. Imaging Features of Invasive and Noninvasive Fungal Sinusitis: A Review, RadioGraphics 2007; 27:1283-1296

8. Hathiram BT, Khattar VS. Fungus ball of paranasal sinuses. Otolaryngol Clin, 2009;1(1):33-35

9. Vyzantiadis TA, Johnson EM, Kibbler CC From the patient to the clinical mycology laboratory: how can we optimize microscopy and culture methods for mould identification? J Clin Pathol 2012; 65:475-483.

10. Pimentel JD. and Lum GD. False-Negative Culture Results with Fungal Isolates from Peritoneal Dialysis Fluid. Clin Microbiol. 2006; 44(3): 1206.

11. Zuluaga A, Ospina-Medina J, Castaño-Gallego I, Arango K, González Á. Frequency of fungal agents identified in sinus samples from patients with clinically suspected rhinosinusitis. Diagn Microbiol Infect Dis. 2015;81(3):208-12.

12. Lamoth F and Alexander BD. Nonmolecular methods for the diagnosis of respiratory fungal infections. Clin Lab Med. 2014;34(2):315-36

13. White PL, Shetty A, Barnes R. Detection of seven Candida species using the Light Cycler system. J Med Microbiology. 2003; 52:229-238.

14. Grosjean P, Weber R. Fungus balls of the paranasal sinuses: a review. Eur Arch Otorhinolaryngol 2007; 264: 461-470

15. deshazo RD, O'Brien M, Chapin K. Criteria for the diagnosis of sinus mycetoma. Allergy Clin Immunol 1997; 7: 475- 486.

16. Winn W, Allen S, Janda. Mycology. Mycological investigations In: Winn WC, Koneman EW, Janda WM, Koneman EW eds. Koneman's Colored Atlas and Textbook of Diagnostic Microbiology, 6th edn. Philadelphia:Lippincott Williams and Wilkins, 2006:1151-1243.

17. Willinger B, Obradovic A, Selitsch B. Detection and Identification of Fungi from Fungus Balls of the Maxillary Sinus by Molecular Techniques. J Clin Microbiol 2003; 41 (2): 581-585

18. Erkan A, Kocü A. Our approach to cases with fungus balls of the paranasal sinuses. J Med Updates. 2014; 4(1):25-28

19. Fanucci E., Nezzo M., Neroni, L. Montesani Jr., Ottria, M. Gargari. Diagnosis and treatment of paranasal sinus fungus ball of odontogenic origin: case report. Oral \& Implantology 2013; 3: $63-66$

20. Giri S, Kindo AJ, Rao S, Kumar AR. Unusual causes of fungal rhinosinusitis: a study from a tertiary care centre in South India. Indian J Med Microbiol 2013; 31(4):379-84.

21. Polzehl D, Weschta M, Podbielski A, Riechelmann H, Rimek $D$. Fungus culture and PCR in nasal lavage samples of patients with chronic rhinosinusitis. J Med Microbiol. 2005; 54:31-7.

22. Jain S, Das S, Gupta N, Malik JN. Frequency of fungal isolation and antifungal susceptibility pattern of the fungal isolates from nasal polyps of chronic rhinosinusitis patients at a tertiary care centre in north India. Med Mycol 2013;51(2):164-9.

23. Prateek S, Banerjee G, Gupta P, Singh M, Goel MM, Verma $V$. Fungal rhinosinusitis: a prospective study in a University hospital of Uttar Pradesh. Indian J Med Microbiol. 2013; 31(3): 266-269

24. Michael RC, Michael JS, Ashbee RH, Mathews MS. Mycological profile of fungal sinusitis: An audit of specimens over a 7-year period in a tertiary care hospital in Tamil Nadu. Indian J Pathol Microbial. 2008; 51(4): 493-496

25. Hajiioannou J, Maraki S, Vlachaki E, Lagoudianakis G, Skoulakis C, et al. Mycology of the Nasal Cavity of Chronic Rhinosinusitis Patients with Nasal Polyps in the Island of Crete. Res Otolaryngol 2012; 1 (2): 6-10

26. Hashemia F, Hashemian F, Bakhshaei M. The prevalence of positive fungal cultures in patients with chronic rhinosinusitis in a high altitude region of iran. Iran J Otorhinolaryngol 2012;24(66):29-33

27. Naghibzadeh B, Razmpa E, Alavi Sh, et al. Prevalence of fungal infection amo.ng Iranian patients with chronic sinusitis. Acta Otorhinolaryngol Ital. 2011;31(1):35-8.

28. Buzina W1, Braun $H$, Freudenschuss $K$, Lackner A, Habermann $W$, et la. Fungal biodiversity as found in nasal mucus. Med Mycol 2003;41(2):149-61.

29. Chatterjee $S$ and Chakrabarti A. Epidemiology and Medical Mycology of Fungal Rhinosinusitis. Otorhinolaryngol Clin 2009;1(1):1-13

30. Lacroix C, Rodier M, Jacquemin J. Detection of Galactomannan for Diagnosis of Fungal Rhinosinusitis. Journal of Clinical Microbiology. 2001;39 (12): 4593-4594

31. Broglie A, Tinguely M, Holzmann D. How to diagnose sinus fungus balls in the paranasal sinus? An analysis of an institution's cases from January 1999 to December 2006. Rhinology. 2009;47: 379-384

32. Patovirta RL, Reiman M, Husman T, Haverinen $U$, Toivola M, Nevalainen A. Mould specific IgG antibodies connected with sinusitis in teachers of mould damaged school: a twoyear follow up study. Int J Occup Med Environ Health. 2003;16(3):221-30.

33. Collins MM, Nair SB, Der-Haroutian V, et al. Effect of using multiple culture media for the diagnosis of noninvasive fungal sinusitis. Am J Rhinol 2005; 9: 41-45. 
34. Kostamo K, Richardson M, Eerola E, etal. Negative impact of Aspergillus galactomannan and DNA detection in the diagnosis of fungal rhinosinusitis. J Med Microbiol 2007; 56: 1322-1327

35. Salmon A., Debourgogne A.,.Vasbien $M$, Clément L, Collomb J, Plénat F, P. Bordigoni1 and Machouart M. Disseminated Scopulariopsis brevicaulis infection in an allogeneic stem cell recipient: case report and review of the literature. Clin Microbiol Infect 2010; 16: 508-512.

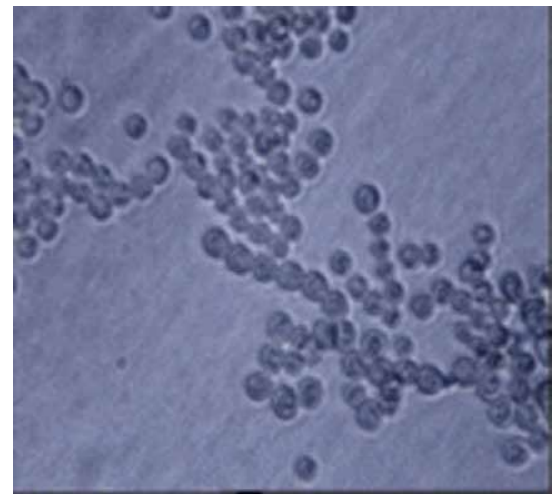

Figure 1. Microscopic appearance (X40) of transparency tape preparation showing growth of scopularopsis.

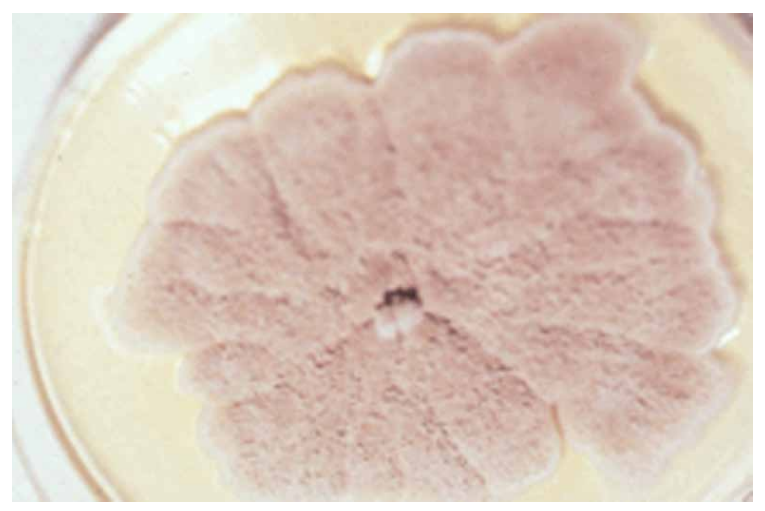

Figure 2. Corn meal agar showing the seven days growth of Scopularopsis colonies.

\section{Comment on this article:}

\section{$8[8$ in $8+S$ P}

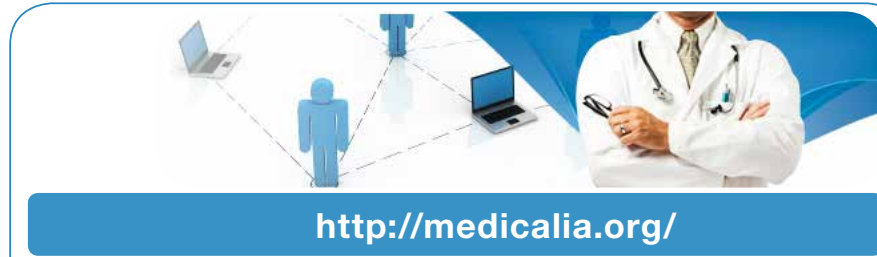

Where Doctors exchange clinical experiences, review their cases and share clinical knowledge. You can also access lots of medical publications for free. Join Now!

\section{Publish with iMedPub}

http://www.imed.pub

The Journal is an open access peer-reviewed journal that publishes scientific papers about all aspects of antimicrobials. The journal will publish original research articles, reviews, brief reports and case reports dealing with basic and clinical antibacterial agents, antiviral, antiprotozoals, antituberculuous, antifungal and antihelminthes agents.

All manuscripts must be prepared in English, and are subject to a rigorous and fair peer-review process. Accepted papers will immediately appear online.

The journal aims to advance the knowledge, attitude and the research of chemotherapy in the Arabic world in cooperation with international, national scientific and public societies as well as research centers with similar aims and objectives. 